\title{
INSTANTANEOUS FLUX MEASUREMENTS USING THE BACKGROUND SIGNAL OF THE RHODIUM SELF-POWERED NEUTRON DETECTOR
}

\author{
S.V. GURU and D.K. WEHE \\ Department of Nuclear Engineering \\ The University of Michigan \\ Ann Arbor, Michigan 48109. U.S.A \\ (Received for publication 26 November 1991)
}

\begin{abstract}
The background current produced by a rhodium self-powered neutron detector (RSPND) with an integral background lead has been investigated to determine its relationship to the neutron flux seen by the detector. The background current and steady state flux were measured at the core midplane for all fuel assemblies, and good linearity was seen. Under transient conditions, the background current, when delayed fission product decay contributions are included, follows the flux reasonably well although with a slight lag. This opens the possibility of using the RSPND during transient conditions.
\end{abstract}

\section{INTRODUCTION}

The rhodium self-powered neutron detector is one of the most common incore detectors used in commercial nuclear power plants. However, because of its delayed dynamic response the signal from this detector is generally useful only at steady state power operations. While there exist self-powered neutron detectors which yield prompt signals, such as cobalt or platinum, they suffer from inferior sensitivity and yield signals which require more frequent calibrations to reactor power or flux. As a result many nuclear power reactors have chosen rhodium as their SPND emitter material and a substantial effort has subsequently been invested into means to improve the RSPND's time response ${ }^{1-3}$. In our work we investigate using the background signal of the detector as a prompt measure of the flux. This method has the potential advantages of being instantaneous, least expensive and can be easily implemented on those RSPNDs which have background leads and are already in operation at various commercial nuclear power plants.

\section{THEORY}

The schematic of a typical RSPND is shown in Figure 1. After a thermal neutron is absorbed by the rhodium emitter, the activated $104 \mathrm{Rh}$ and $104 \mathrm{mRh}$ decay primarily through the 
emission of energetic $\beta$ particles. Some of these $\beta$ particles escape from the rhodium emitter and contribute to a measured signal current. Since this current is proportional to the neutron absorption rate by the rhodium emitter, the current produced is proportional to the neutron flux at steady state. A background lead of length equal to that of the signal lead is used to measure the background current. The background current is an integral measurement of current generating interactions taking place over the entire length of the background lead. This background lead is subjected to the same interactions as the signal lead and hence measuring the currents from the background and emitter leads and taking the algebraic difference gives the net current due to reactions in the emitter alone.

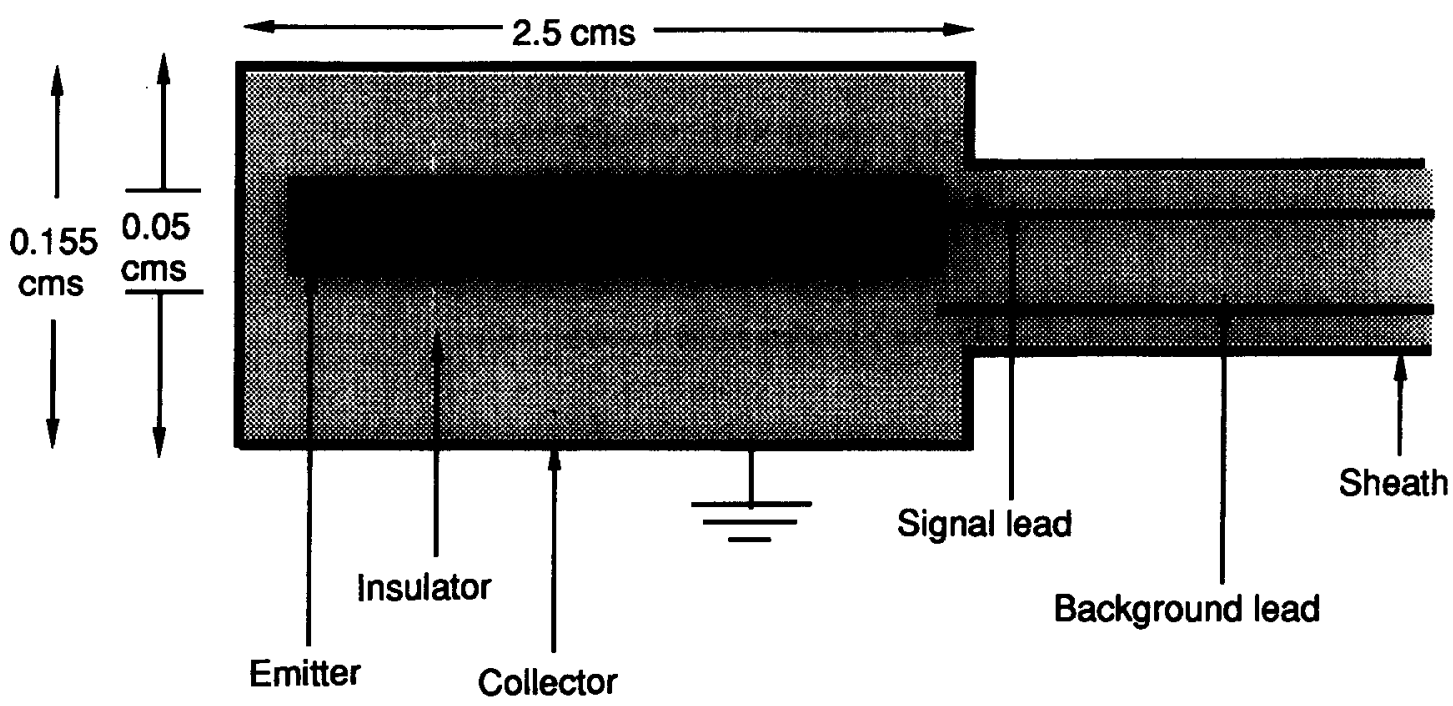

Fig. 1. Schematic of a Rhodium Self-Powered Neutron Detector

The primary component of the integral background current of the RSPND comes from the core gamma rays undergoing Compton scattering from the cable wire, insulation, or the Inconel sheath and creating free Compton electrons. Under steady state conditions we expect the integral background current to be directly proportional to the neutron flux:

$$
I_{p}=c_{1} \phi+c_{2}
$$

where $c_{1}$ and $c_{2}$ are assumed only weakly time dependent and must be determined from prior experiments. Note that equation (1) is only an hypothesis and must be tested experimentally.

\subsection{Delaved fission product decav contribution to the hackground signal}

Equation (1) accounts for the background current when the neutron and gamma flux do not vary with time. However, under that condition, the usual RSPND signal provides an accurate measure of the flux anyway. If the flux level varies with time then the background current of the RSPND consists of two terms, the prompt $I_{p}$ and the delayed component $I_{d}$. The delayed 
component is induced by the delayed fission product decays. The background current $\mathrm{Ib}(\mathrm{t})$ is written as:

$$
I_{b}(t)=I_{p}(t)+I_{d}(t)
$$

The delayed background signal $I_{d}(t)$ due to previous fissions can be approximated by:

$$
I_{d}(t)=c \int_{-\infty}^{t} \lambda \Sigma_{f} \phi\left(t^{\prime}\right) e^{-\lambda\left(t-t^{\prime}\right)} d t^{\prime}
$$

Taking the derivative of equation (3) with respect to time, one obtains:

$$
\frac{d I_{d}}{d t}+\lambda I_{d}(t)=c \lambda \Sigma_{f} \phi(t)
$$

Since only the total background current is observed equation (4) is not useful by itself. Instead we multiply equation (1) for the prompt background by $\lambda$ and to this we add the derivative of equation (1) with respect to time to give:

$$
\frac{d I_{p}}{d t}+\lambda I_{p}(t)=c_{1} \lambda \phi+c_{2} \lambda+c_{1} \frac{d \phi}{d t}
$$

Adding equations (4) and (5) yields:

$$
\frac{d I_{b}}{d t}+\lambda I_{b}(t)=c \lambda \Sigma_{f} \phi(t)+c_{1} \lambda \phi(t)+c_{1} \frac{d \phi}{d t}+c_{2} \lambda
$$

Assuming an exponential variation in flux, we have:

$$
\frac{d \phi}{d t}=k(t) \phi(t)
$$

where $k(t)$ is an estimable parameter whose value at steady state is zero. This approximation will be valid provided the measurements are made on a time sale small compared to significant changes in the flux. Making this substitution into equation (6) gives:

$$
\mathrm{I}_{\mathrm{b}}(\mathrm{t})+\frac{1}{\lambda} \frac{\mathrm{dI}}{\mathrm{dt}}=\left[\mathrm{c}_{1}+\mathrm{c}_{1} \frac{\mathrm{k}}{\lambda}+\mathrm{c} \Sigma_{\mathrm{f}}\right] \phi+\mathrm{c}_{2}
$$

The term $c_{1}+c \sum_{f}$ can be identified as the value actually measured as $c_{1}$ in a steady state experiment. Defining this term $c_{1}$ ', we obtain:

$$
\mathrm{I}_{\mathrm{b}}(\mathrm{t})+\frac{1}{\lambda} \frac{\mathrm{d} \mathrm{I}_{\mathrm{b}}}{\mathrm{dt}}=\mathrm{c}_{1}{ }^{\prime}\left\{1+\left[1-\frac{\mathrm{c} \Sigma_{\mathrm{f}}}{\mathrm{c}_{1}{ }^{\prime}}\right] \frac{\mathbf{k}}{\lambda}\right\} \phi+\mathrm{c}_{2}
$$


Note that, in general, equation (9) requires knowledge of $k(t), \lambda, c_{1}{ }^{\prime}, c_{2}$ and $c \Sigma_{f}$. We have also implicitly assumed that a single average value of $\lambda$ can be applied. To get a feel for the relative sizes of these terms, we note that, at steady state, for a typical reactor, $I_{d} \approx 1 / 3 I_{b}$. Then using equation (4)

$$
\frac{I_{d}}{I_{b}-c_{2}}=\frac{c \Sigma_{f}}{c_{1}^{\prime}}
$$

Noting that $c_{2}$ is small compared to $I_{b}$, implies:

$$
\frac{c \Sigma_{f}}{c_{1}{ }^{\prime}} \approx \frac{1}{3}
$$

Thus the term in square brackets in equation (9) can not be neglected. However, recognizing

$$
k(t)=\frac{\partial}{\partial t}[\ln \phi(t)]=\frac{1}{T(t)}
$$

where $\mathrm{T}$ is the reactor period, for reasonably long reactor periods compared to $\lambda$, equation (9) will reduce to :

$$
\mathrm{I}_{\mathrm{b}}(\mathrm{t})+\frac{1}{\lambda} \frac{\mathrm{dI}}{\mathrm{dt}}=\mathrm{c}_{1}{ }^{\prime} \phi+\mathrm{c}_{2}
$$

Thus, equation (10) should be valid for cases where $\lambda \mathrm{T} \gg 1$. During a rapid flux change, one would expect $\lambda$ to be relatively large due to the enhanced contributions of the short lived fission products, but $T$ will be smaller. Thus, it is difficult to predict if and when equation (10) will hold during a transient and we must turn our attention to benchmark experiments. Of course, after a flux change as $T \rightarrow \infty$, we would expect equation (10) to be valid. Note that equation (10) is similar to equation (1) except that the addition of the derivative term accounts for the lag in the total background signal induced by radioactive decays.

\section{EXPERIMENTAL RESULTS}

The RSPND used in these experiments is shown in Figure 1. The emitter is $25 \mathrm{~mm}$ in length and $0.5 \mathrm{~mm}$ in diameter. The lead and background cables are identical and are made of Inconel $600,0.25 \mathrm{~mm}$ in diameter and $\mathrm{MgO}$ is used for the insulator. Two sets of experiments were performed in the core of the Ford Nuclear Reactor (FNR) in a thermal flux of $\approx 10^{13} \mathrm{n} / \mathrm{cm}^{2}-\mathrm{s}$. Figure 2 gives the schematic of the FNR core for reference.

\subsection{Steady state experiments}

In the first set of experiments the reactor was maintained at a steady power and the RSPND was moved to different core locations to measure the signal and the background current from the detector. The background current was measured instantaneously while the signal current 
was measured after waiting for 20 minutes. These measurements were done at every core element shown in Figure 2 with the detector inserted 12" (core midplane) into each element. Due to the constant flux levels during each measurement, the total background current measured is from the steady state values of the prompt and delayed components. These measurements test the validity of equation (1) throughout the core, and determine $c_{1}$ ' and $c_{2}$.

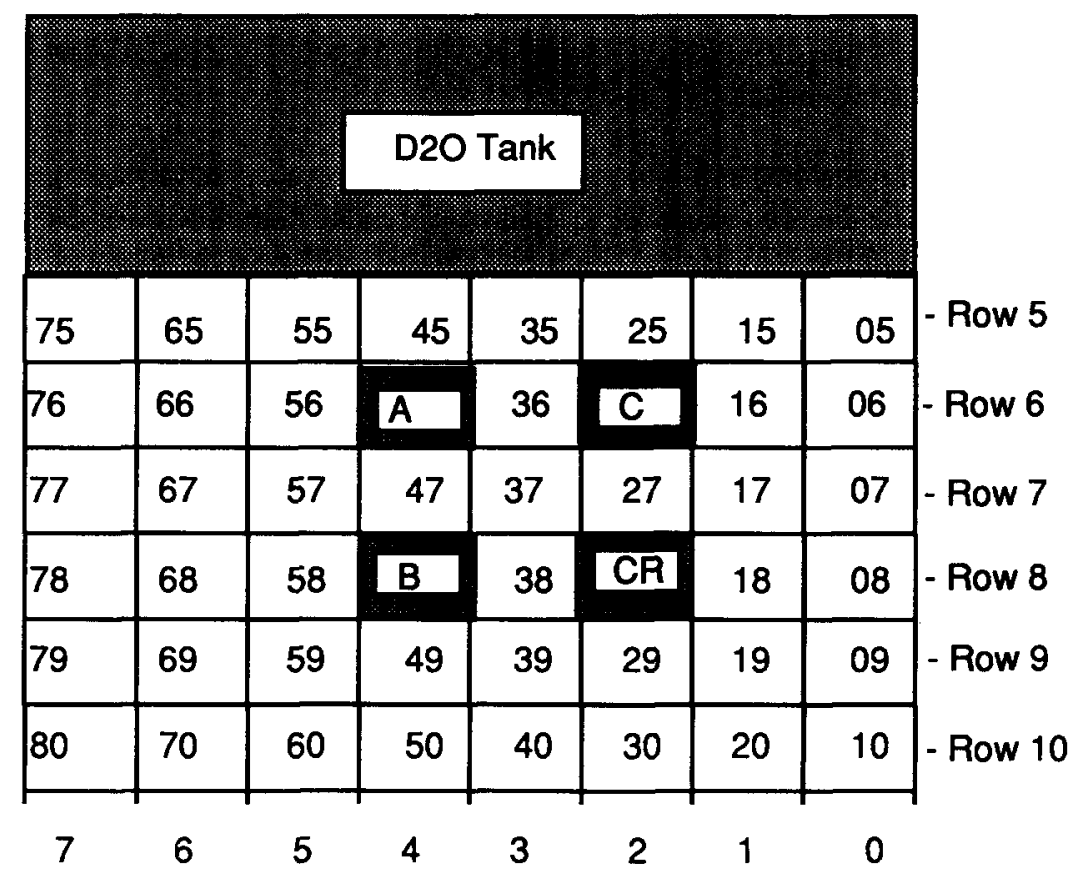

Column numbers

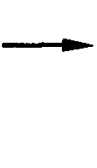

Fig. 2. Core layout and fuel element numbering at the FNR.

$\mathrm{A}, \mathrm{B}, \mathrm{C}$ and $\mathrm{CR}$ represent modified fuel assemblies which accept control rods.

Noting that

$$
k(t)=\frac{d I_{b}}{d t}=0
$$

equation (9) becomes:

$$
I_{b}(\underline{r})=c_{1}^{\prime}(\underline{r}) \phi(\underline{r})+c_{2}(\underline{r})
$$

Thus, knowing $\phi(r)$ from a measurement of the net RSPND signal, and $I_{b}(r)$ from the background signal, $c_{1}{ }^{\prime}(r)$ and $c_{2}(r)$ can be determined. 
Figure 3(a) plots the absolute value of the background current and the measured subcadmium flux along the various elements in row 8 . Note the generally good agreement. Figure 3(b) plots the background current versus the subcadmium flux. The slope is $\mathrm{c}_{1}$ ' and the intercept gives $\mathrm{c}_{2}$. Figures 3(a) and (b) pertain to measurements made along row 8, but similar results were obtained for all of the rows shown in Figure 2. Thus, $c_{1}{ }^{\prime}(r)$ and $c_{2}(r)$ were measured for every element at the core midplane. In addition data were acquired over three different fuel cycles to test whether the measured values varied for different core loadings. The constants $\mathrm{c}_{1}$ ' and $\mathrm{c}_{2}$, averaged over the three different cycles are shown in Figures 4 and 5 . A least squares fit was used to estimate the uncertainties in $c_{1}$ ' and $c_{2}$.

Figures 4(a) and 5(a) give the average values $c_{1}$ ' and $c_{2}$ along various rows within the FNR core. The average values of $c_{1}$ ' and $c_{2}$ were also determined for each column within the FNR core for the three fuel cycles. Figures 4(b) and 5(b) give the average values of $c_{1}$ ' and $c_{2}$ along various columns within the FNR core.

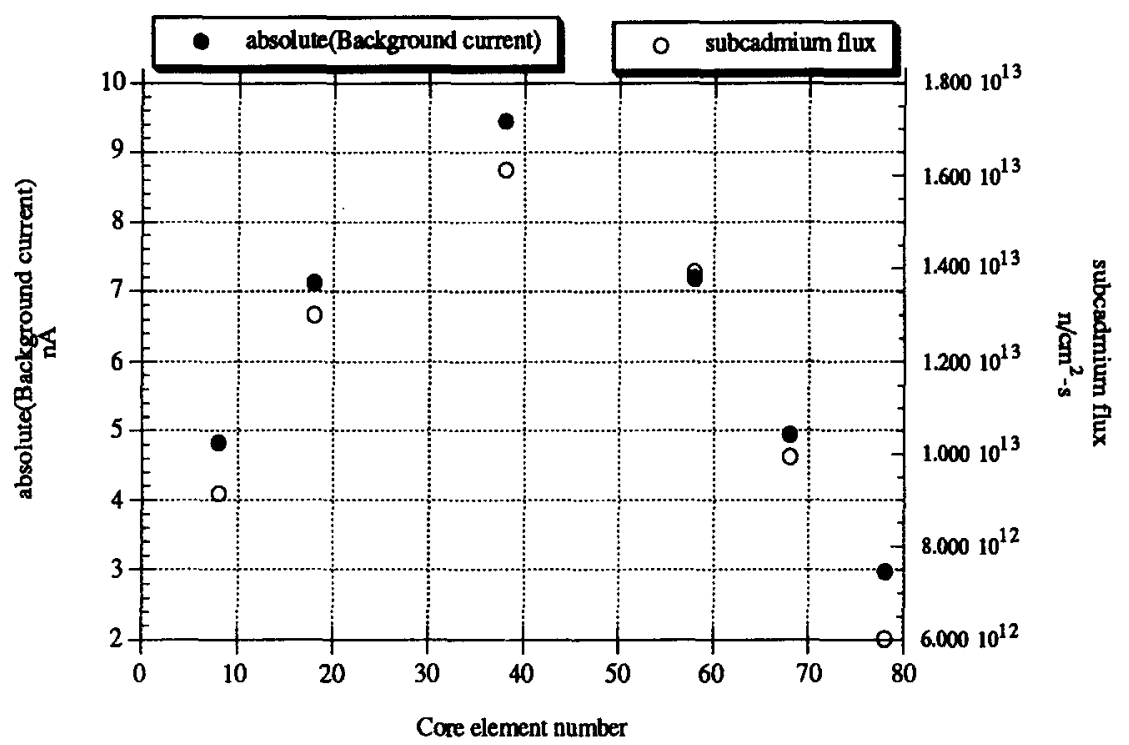

Fig. 3(a). The absolute value of background current and the subcadmium flux are shown for different elements across row 8. Measurements were done with the RSPND at core midplane.

From Figures 4(a) and 5(a), $c_{1}$ ' appears to increase near the light water reflector while $c_{2}$ tends to decrease. Significantly higher values of $c_{1}$ ' and lower values of $c_{2}$ are observed for rows 9 and 10. The column-averaged values of $c_{1}$ ' and $c_{2}$ shown in Figures 4(b) and 5(b) also indicate an increase in the value of $c_{1}$ ' as we move outwards towards one reflector, yet show a surprising decrease near the other reflector. The smallest column-averaged value of $\mathrm{c}_{1}$ ' was recorded at the center of the core (for column number 3 ) and the value increased slightly for the columns on either direction. The largest column-averaged value of $c_{2}$ was recorded at column 3 
and it decreased slightly for columns on either side of it. The behavior of column 0 is surprising, and inconsistent with other data near the light water reflector.

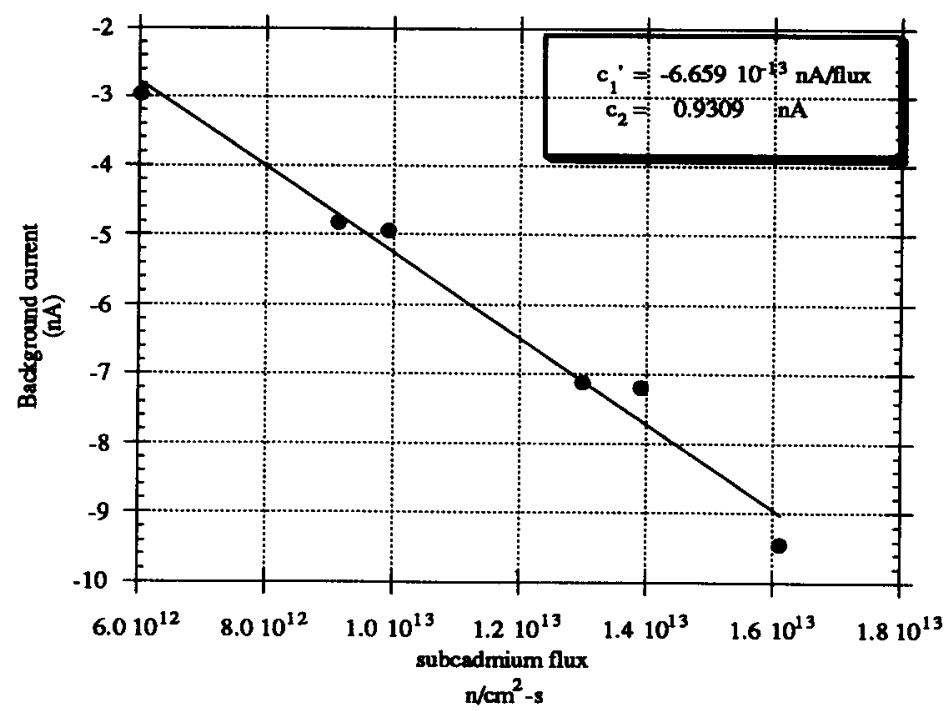

Fig.3(b). Relationship between background current and the measured subcadmium flux

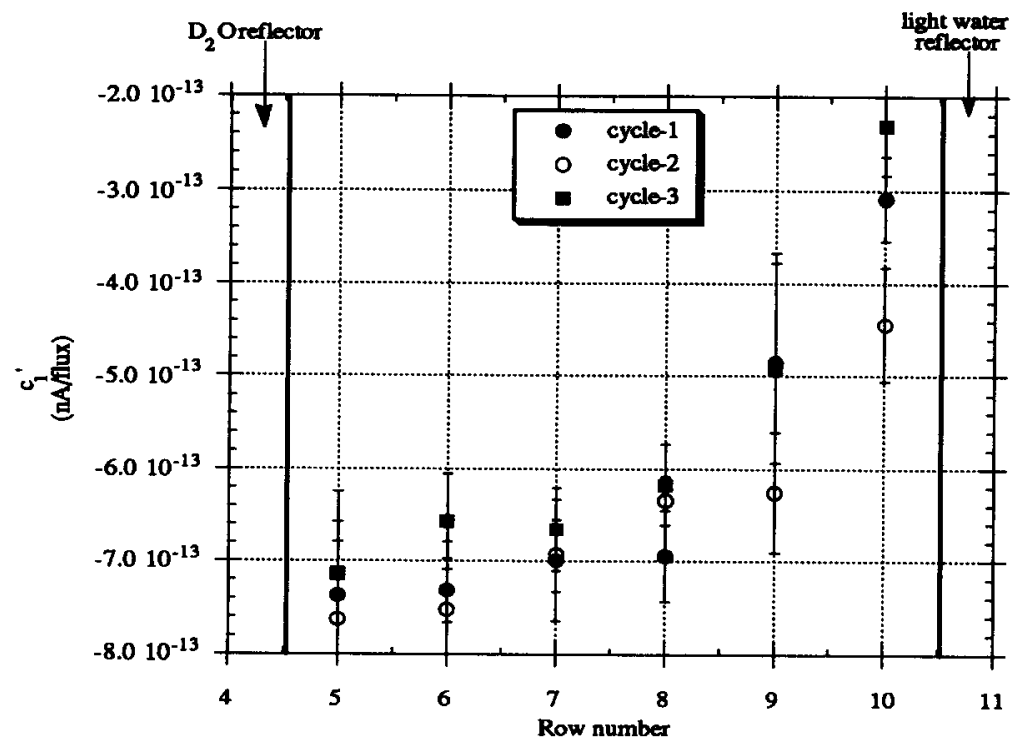

Fig. 4(a). Average values of $c_{1}$ ' for different rows within the FNR. 


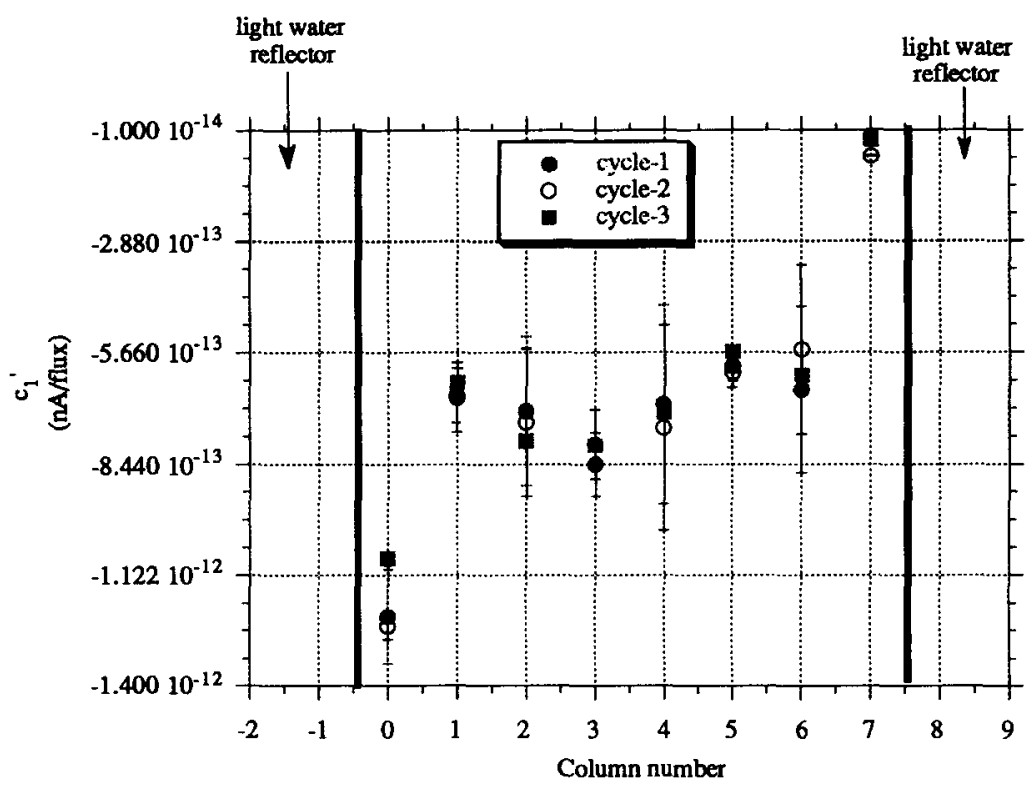

Fig. 4(b). Average values of $c_{1}{ }^{\prime}$ for different columns within the FNR core.

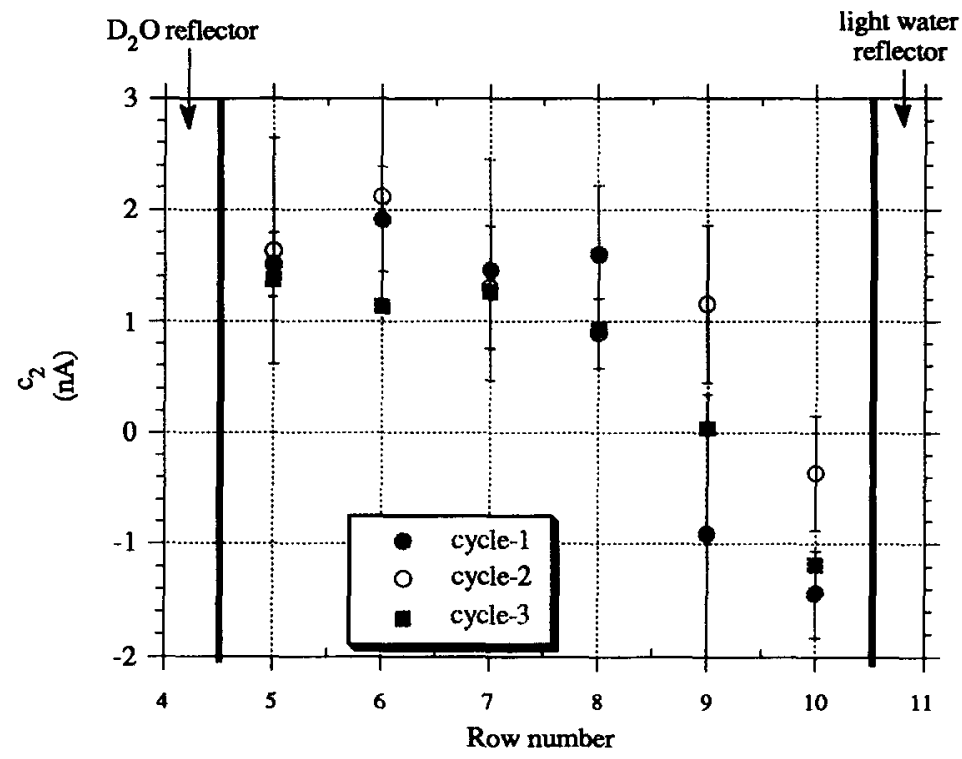

Fig. 5(a). Average values of $c_{2}$ for different rows within the FNR.

The variations of $c_{1}$ ' and $c_{2}$ near the reflector may be attributable to the change in the ratio of the thermal to epithermal flux, near the outer core elements. The subcadmium flux $\phi_{\mathrm{Sc}}$, is calculated from the net RSPND current, $I_{\text {net }}$, as: 


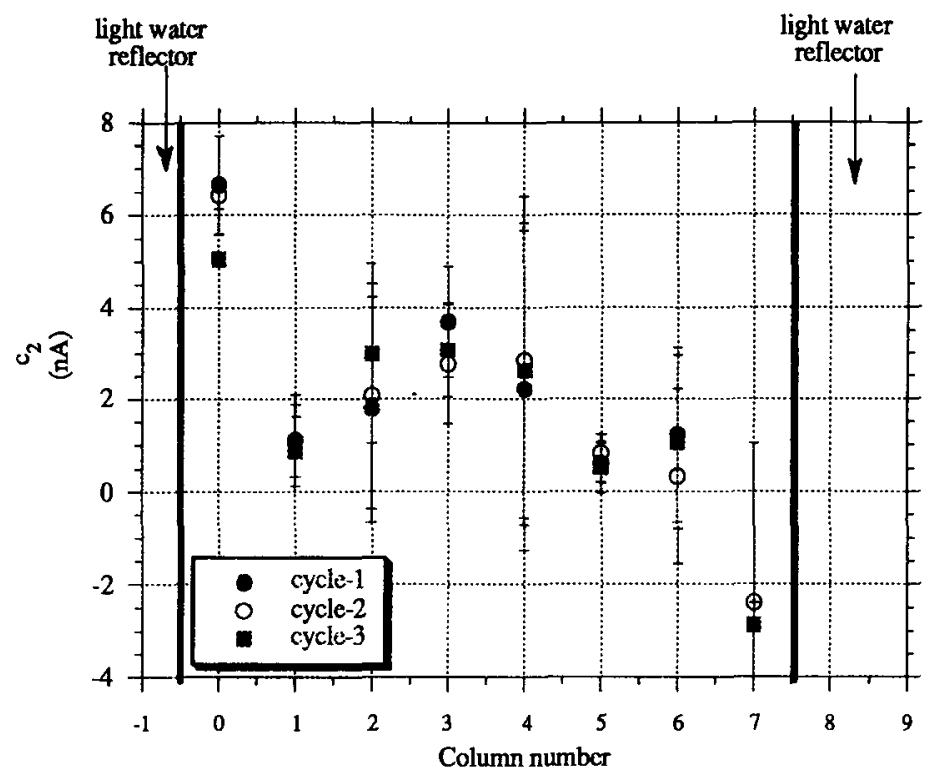

Fig. 5(b). Average values of $c_{2}$ for different columns within the FNR

$$
\phi_{\mathrm{sc}}=\frac{\mathrm{f}_{\mathrm{sc}} \mathrm{I}_{\text {net }}}{\mathrm{S}_{\mathrm{sc}}}
$$

where $f_{S c}$ is the fraction of the net current induced by subcadmium neutrons, and $S_{S c}$ is the sensitivity of the detector to subcadmium neutrons. In our analysis, we have used constant values of $f_{S c}$ and $S_{S c}$ throughout the core. Since rhodium wire activations have shown $f_{S c}$ to increase by several percent near the reflectors, our assumption would lead to an underprediction of $\phi_{\mathrm{Sc}}$ and thus overpredicted values of $c_{1}$ '. It is significant, however, that throughout the majority of the core, the values of $c_{1}$ ' and $c_{2}$ are reasonably constant.

With the midplane values of $c_{1}{ }^{\prime}$ and $c_{2}$ determined, the flux was predicted at a later time from the background current alone. The detector was placed at the core midplane and the background was measured in the elements of row 7 . The reactor was in an equilibrium full power configuration. Figures $6(a)$ and (b) show the results of these experiments. The average values of $c_{1}$ and $c_{2}$ determined for row 7 have been used to predict the flux in Figure 6(a) and the average values of $c_{1}$ ' and $c_{2}$ determined for columns 0 to 6 have been used to predict the flux in Figure 6(b). Note the uncertainty in the instantaneous background-predicted flux is estimated to be rather large compared to the net signal flux, as expected. 


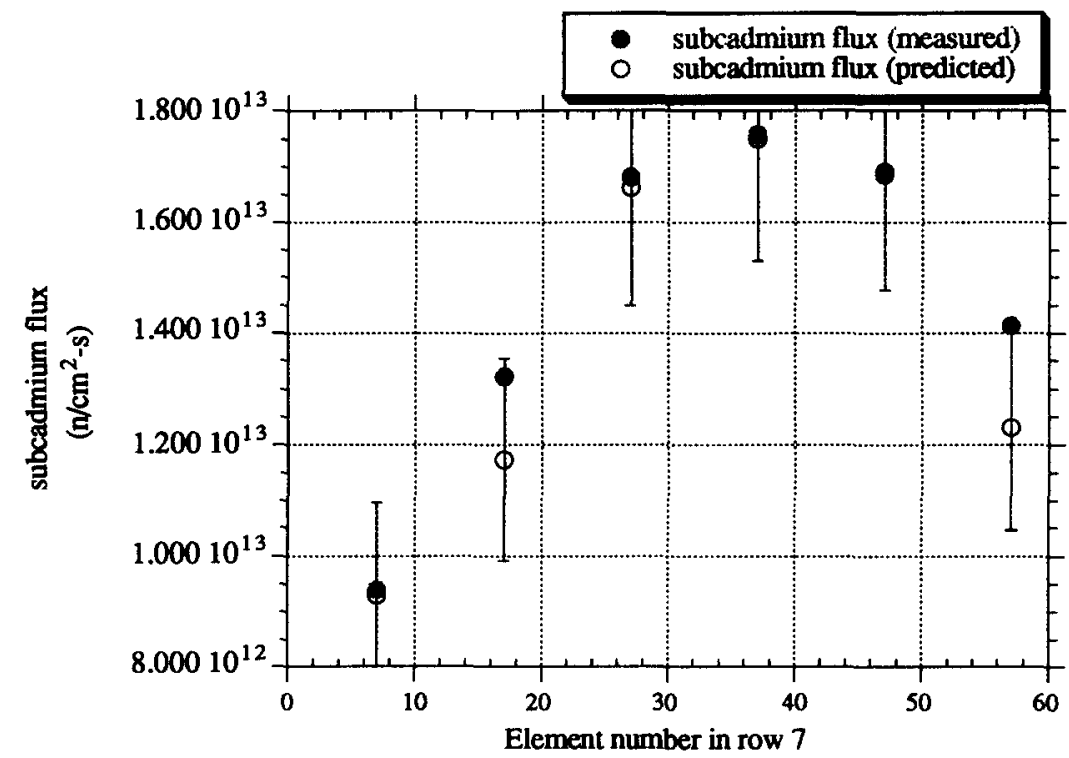

Fig. 6(a). Comparison of the subcadmium flux predicted in a steady flux field by delayed net signal measurement and instantaneous background measurement. The values of $c_{1}{ }^{\prime}$ and $c_{2}$ used here were determined for row 7.

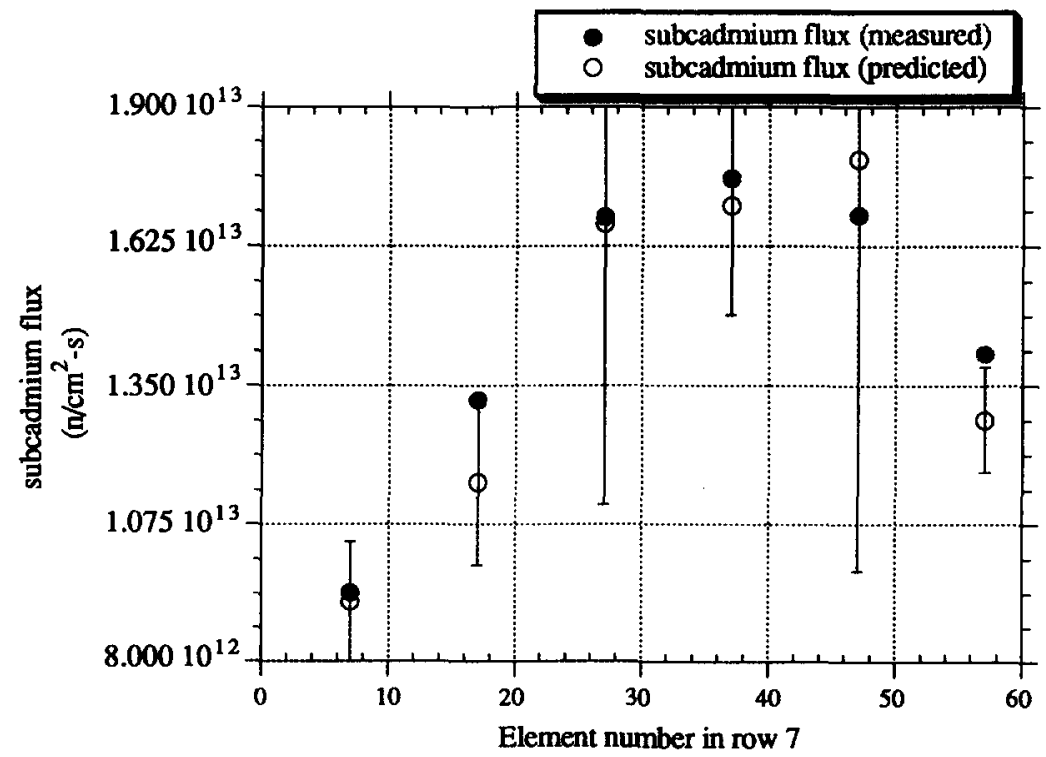

Fig. 6(b). Comparison of the subcadmium flux predicted in a steady flux field by delayed net signal measurement and instantaneous background measurement. The values of $c_{1}$ ' and $c_{2}$ used here were determined from column 0 to column 6. 


\subsection{Dynamic Flux Experiments}

The second set of experiments consisted of positioning the detector at a fixed location and varying the flux. It is under this condition where we hope the background signal can provide some dynamic flux information since the net signal is not useful. The location chosen was near the core center (element 37) and the detector was placed at the core midplane. This location was chosen since it had the highest flux level and produced reasonable background currents. The detector was then located at a fixed position and the power of the reactor was varied. The reactor was taken to different power levels and was maintained at each power level for a period of 30 minutes. The background current was measured instantaneously at the new point while the signal current was measured after 20 minutes. Using the values of $c_{1}{ }^{\prime}$ and $c_{2}$ determined in Figures 4 and 5 and an experimentally measured average decay constant $\lambda\left(1.008 \times 10^{-4} \mathrm{sec}^{-1}\right)$, the flux was predicted using equation (10). This experimentally measured average decay constant $\lambda$ was determined earlier by plotting the background current against time for a long period of time following a change in the flux level. The predicted flux and the measured flux are shown in Figure 7. Note that this method consistently overpredicts the flux measured, with an average deviation of $5 \%$ and a maximum deviation of $15 \%$. This agreement could be improved by using a slightly smaller value of $\lambda$.

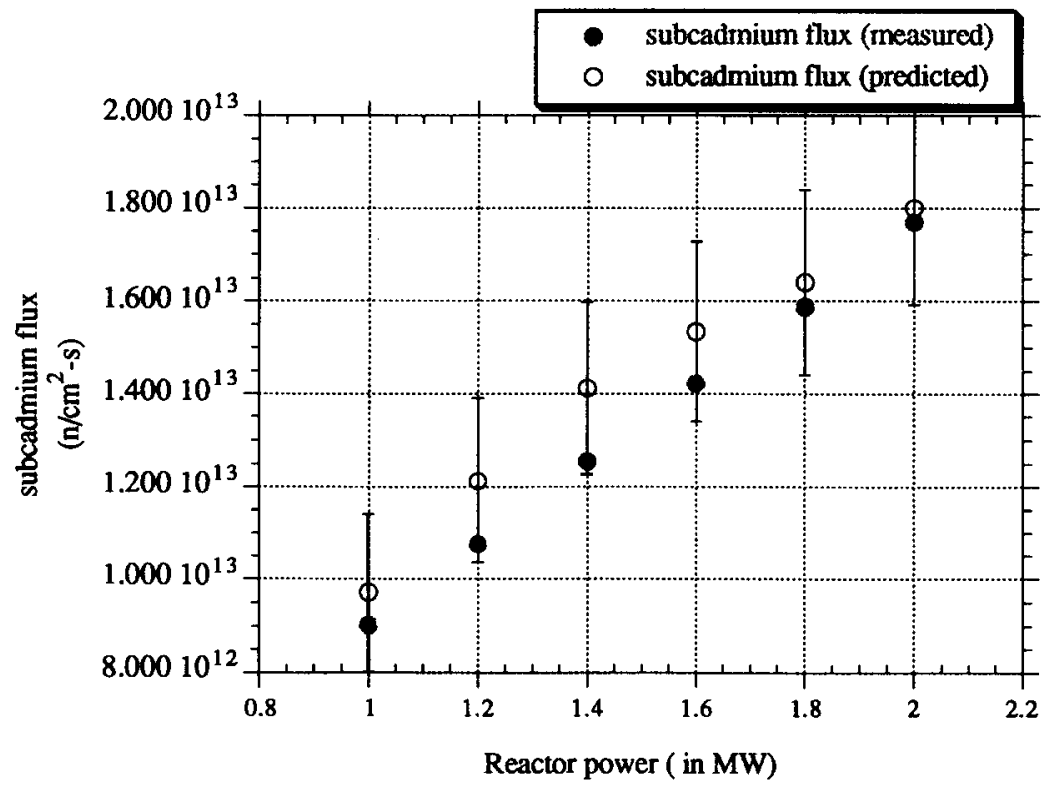

Fig.7. Comparison of the subcadmium flux predicted in a varying flux field by delayed net signal measurement and instantaneous background measurement. 


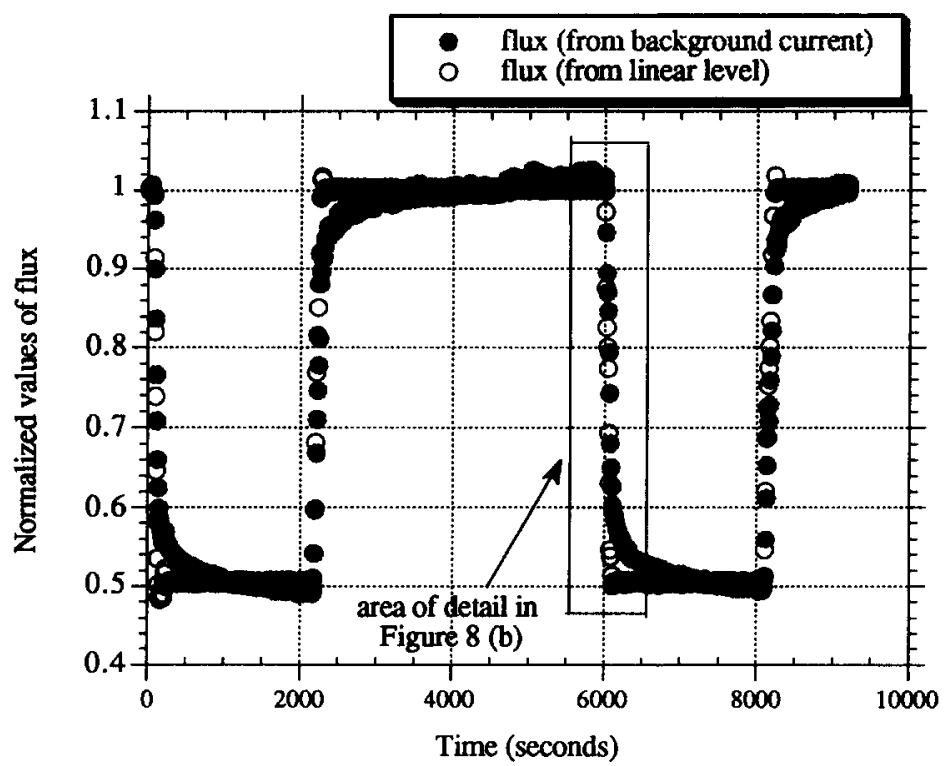

Fig. 8(a). Comparison of the flux measured by the background current and by the linear level.

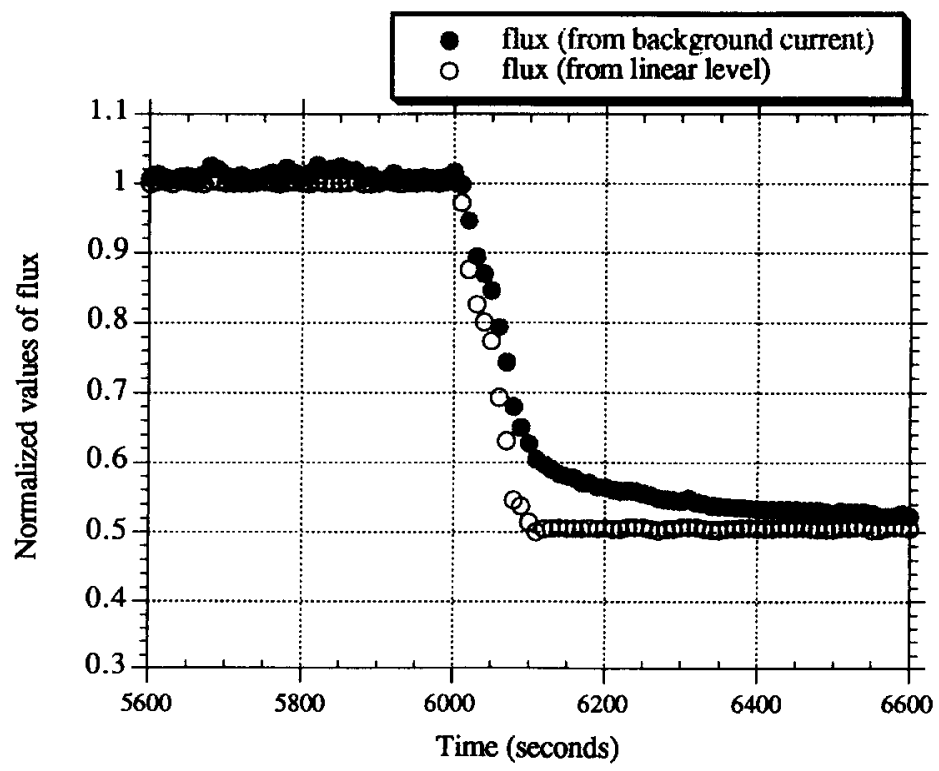

Fig. 8(b). Region of interest selected in Fig. 8(a).

Comparison of the flux measured from the background current and from the linear level during a flux transient. 
In a more demanding experiment, the reactor was fluctuated between full and $50 \%$ power levels and the RSPND background signal was recorded at the rate of $1 \mathrm{~Hz}$ using a data acquisition system. The data from an excore compensated ion chamber (the linear level) was also recorded to serve as a comparison to the flux level measured from the background current. The flux was again predicted from the knowledge of the background current only and the result is shown in Figures 8(a) and (b). All the acquired signals were normalized to their values at steadystate full power of the reactor.

We have deliberately chosen a higher value $\left(1.25 \mathrm{sec}^{-1}\right)$ for the average decay constant $\lambda$ than the smaller value measured experimentally from the background data. The smaller value of $\lambda$ leads to large oscillations in the recovered flux due to the $1 / \lambda$ term multiplying $\mathrm{dI} / \mathrm{dt}$. As a result, the background lags the flux in Figures 8 (a) and (b). Note, however, that the rapid rises and falls of the flux are mapped fairly well. At this high sampling rate, the shorter lived fission products contribute significantly to the initial changes in the background signal measured, and justify the higher value of $\lambda$.

\section{CONCLUSION}

In this work we have explored the idea of using the integral RSPND background signal as a means of determining the flux during a transient. Since the net detector current is delayed, this background signal allows the RSPND to be useful during flux changes. Under static-equilibrium conditions, $c_{1}{ }^{\prime}(r)$ and $c_{2}(r)$ seem to be fairly constant within the core and gave reasonable predictions of the flux. Under transient conditions, the technique also worked reasonably well, although the $(1 / \lambda \times \mathrm{dIb} / \mathrm{dt})$ term was problematic. Since $\lambda$ is small and $\mathrm{dI} b / \mathrm{dt}$ is noisy at sampling rates $\geq 1 \mathrm{~Hz}$, an artificially enlarged value of $\lambda$ was used which led to a slight lag in the predicted flux. Nevertheless, the results are very encouraging since they demonstrate a technique by which the RSPND may be made useful during flux changes.

\section{ACKNOWLEDGEMENTS}

The authors would like to acknowledge the generous assistance of Mr. David O'Connor in performing the static field experiments, Prof. John Lee for his guidance, and Mr. Siaka Yusuf for his contributions during the initial part of this work.

\section{REFERENCES}

1. Kantrowitz M.L. (1987) IEEE Trans, on Nucl. Sci., NS-34, 1.

2. Rombough C.T. et.al. (1989) Trans. Am. Nucl. Soc., 59, 338.

3. Yusuf S.O. and Wehe D.K. (1989) Trans. Am. Nucl. Soc., 59, 336. 